\title{
Optimal Cerebral Perfusion Pressure in Centers with Different Treatment Protocols
}

Tim Howells, PhD; Peter Smielewski, PhD; Joseph Donnelly, MD; Marek Czosnyka, PhD; Peter J.A. Hutchinson, PhD, FRCS (SN), FMedSci; David K. Menon, MD, PhD, Per Enblad, MD, PhD, Marcel J.H.. Aries, MD

From: Department of Neuroscience, Section of Neurosurgery (TH, PE), Uppsala University Hospital, Sweden; Department of Clinical Neurosciences, Neurosurgical Unit (PS, JD, MC, PJAH), Division of Anaesthesia (DKM), University of Cambridge, Addenbrooke's Hospital, Cambridge, UK; Department of Critical Care (MJHA), Maastricht University Medical Center, Maastricht University, The Netherlands; Institute of Electronic Systems (MC), Warsaw University of Technology, Poland

Conflicts of interests: Dr. Czosnyka and his institution received licensing fees for the ICM+ software, as did Dr. Smielewski and his institution. The remaining authors have declared that they do not have any potential conflicts of interest.

Funding: Dr. Menon is supported by the National Institute for Health Research (NIHR), UK, through a Senior Investigator Award and funding provided to the NIHR Cambridge Biomedical Centre; and by the European Union through a FP-7 grant for the CENTER-TBI Study (Agreement Number: 602150). Dr. Hutchinson is supported by a NIHR Research Professorship and the NIHR Cambridge BRC.

Correspondence to: Tim Howells, Department of Neuroscience, Section of Neurosurgery, Uppsala University Hospital, Sweden; email: timothy.howells@lul.se; phone: +46 18 6114971; fax: 018-558617 


\section{Abstract}

Objectives: The three centers in this study have different policies regarding cerebral perfusion pressure (CPP) targets and use of vasopressors in traumatic brain injury (TBI) patients. The aim was to determine if the different policies affected the estimation of CPP which optimizes the strength of cerebral autoregulation, termed CPPopt.

Design: Retrospective analysis of prospectively collected data.

Setting: Three neurocritical care units at university hospitals in Cambridge, UK, Groningen, the Netherlands, and Uppsala, Sweden.

Patients: A total of 104 TBI patients were included: 35 each from Cambridge and Groningen, and 34 from Uppsala.

Interventions: None.

Measurements and Main Results: In Groningen the CPP target was $\geq 50$ and $<70 \mathrm{mmHg}$, in Uppsala $\geq 60$, and in Cambridge $\geq 60$ or preferably $\geq 70$. Despite protocol differences median CPP for each center was above $70 \mathrm{mmHg}$. CPPopt was calculated as previously published and implemented in the ICM+ software by the Cambridge group, now replicated in the Odin software in Uppsala. Periods with CPP above and below CPPopt were analyzed, as were absolute difference between CPP and CPPopt and percentage of monitoring time with a valid CPPopt. Uppsala had the highest CPP/CPPopt difference. Uppsala patients were older than the other centers, and age is positively correlated with CPP/CPPopt difference. CPPopt was significantly lower in Groningen than in Cambridge. There were no significant differences in percentage of monitoring time with valid CPPopt. Summary CPPopt curves were generated for the combined patient data for each center. These summary curves could be generated for Groningen and Cambridge, but not Uppsala. The older age of the Uppsala patient cohort may explain the 
absence of a summary curve.

Conclusions: Differences in CPPopt calculation were found between centers due to demographics (age) and treatment (CPP targets). These factors should be considered in the design of trials to determine the efficacy of autoregulation guided treatment.

Keywords: Cerebral Perfusion Pressure, Optimal Cerebral Perfusion Pressure, Traumatic Brain Injury, Intracranial Pressure, Blood Pressure, Cerebral Blood Flow, Treatment Protocols 
The management of cerebral perfusion pressure (CPP) in traumatic brain injury (TBI) patients varies in different neurosurgical centers due to inconclusive and conflicting evidence regarding the effects of pharmacologically adjusting CPP. In 1996 [1] and again in 2000 [2] the Brain Trauma Foundation (BTF) suggested maintaining CPP over $70 \mathrm{mmHg}$ as a treatment “option”, which was their weakest recommendation level. In 2007 [3] the BTF revised the recommended CPP range to 50 to $70 \mathrm{mmHg}$, so that the previous lower-limit became an upper-limit. This change was motivated primarily by concerns about the possible negative systemic effects of vasopressors [4]. Recently, in 2016 [5], the recommendation was again changed to either 60 or $70 \mathrm{mmHg}$ as a lower-limit depending on the individual patient. Because this change was based on a single-center study from Cambridge [6], the BTF added the caveat that the specific TBI treatment protocol at that site may have influenced the results. This is a significant point, since a study involving two centers (Edinburgh and Uppsala) with different CPP treatment strategies found that the differences led to radically different relationships between CPP levels and functional outcome [7]. In the center practicing aggressive CPP management, CPP below 70 mmHg was strongly associated with poor outcome, but in the other center lower CPP (50 - 60 mmHg) was associated with good outcome. Both the 2007 and 2016 editions of the BTF Guidelines emphasized the importance of considering a patient's autoregulation status in determining the appropriate individual CPP level, but did not specify a method.

A promising approach is to optimize CPP (CPPopt) [8 - 11] individually and continuously based on the pressure reactivity index (PRx). PRx is computed as the correlation of the slow waves of mean arterial pressure (MAP) and intracranial pressure (ICP), and is well validated as an index of cerebrovascular pressure reactivity in severe TBI patients [12 - 14]. As such it is a good surrogate for the general health, responsiveness and functional integrity of the 
cerebral vessels. Since PRx and CPPopt are computed using only the ICP and MAP waveforms, they can be made available continuously as part of routine monitoring. In retrospective TBI studies divergence from CPPopt has been found to be associated with unfavorable outcome [8, 9].

The aim of this observational study was to determine the effects of treatment differences on the estimation of CPPopt in three academic neurosurgical centers with differing CPP treatment protocols, and to determine the extent to which the protocols are adhered to in clinical practice.

\section{Materials and Methods}

\section{Patient Material}

A total of 104 TBI patients admitted to our neurosurgical units between May 1, 2012 and April 31, 2015 were included: 35 each from Cambridge and Groningen, and 34 from Uppsala. The only requirement was at least 6 hours of continuous computerized ICP and MAP data collected during the first four days of intensive care. Admission and outcome characteristics for the three cohorts are summarized in table 1 , (rows $1-4$ ). Clinical status on admission was assessed using the Glasgow Coma Scale (GCS), and outcome at 6 months using the Glasgow Outcome Scale (GOS) by outpatient or telephone interview. Computerized Tomography (CT) scans were used to classify injuries as focal or diffuse.

\section{Physiological Monitoring}

ICP was monitored in Cambridge using an intraparenchymal sensor (Codman Microsensor ${ }^{\mathrm{TM}}$ ). In Groningen an intraventricular sensor system with optional CSF drainage (Raumedic Neurovent ${ }^{\mathrm{TM}}$ ) was used in all patients. In Uppsala either an EVD system (Smith Medical Hanni- 
Set ${ }^{\mathrm{TM}}$ pressure transducer) or the Codman was used. Twenty Uppsala patients were monitored using an intraparenchymal sensor, 6 with an EVD, and 8 with both.

Physiological data were acquired using the Intensive Care Monitoring $\left(\mathrm{ICM}+{ }^{\circledR}\right)$ software [15] in Cambridge and Groningen, and the Odin Software [7] in Uppsala. All parameters reported were calculated within the first four days of monitoring in intensive care. All patients had more than 7 hours of CPP data during this period, with a median duration of CPP monitoring of 85 hours and interquartile range (IQR) of 68 to 93 hours.

CPP was measured similarly in the three centers. Blood pressure was zeroed at the level of the right atrium. If possible the head was elevated at an angle of $30^{\circ}$ in Uppsala, and $30-45^{\circ}$ in Cambridge and Groningen. CPPopt was calculated according to the algorithm described by Aries et al. [9].

\section{Local Treatment Protocol Differences}

Cambridge [16] and Uppsala [17] have published their protocols. All three centers tried to keep ICP $<20$ mmHg. Cambridge aimed to maintain CPP $\geq 60$ or preferably $\geq 70$, Groningen's goal was to keep CPP between 50 and 70, and Uppsala CPP $\geq 60$, or $\geq 50 \mathrm{mmHg}$ in the case of thiopental treatment. CPPopt curves were available in Cambridge on bedside computers, but there were no specific CPPopt guidelines.

Cambridge and Groningen used vasopressors (mainly norepinephrine) and fluids to control MAP and CPP. Uppsala largely avoided the use of vasopressors, and when used it was to treat MAP. Unlike Uppsala and Groningen, Cambridge added neuromuscular blocking agents (atracurium) as part of the first-tier treatment for intracranial hypertension. 
In Cambridge and Groningen sedation and analgesia was based on the use of opiates (fentanyl or morphine), propofol (maximum $4 \mathrm{mg} / \mathrm{kg} / \mathrm{hr}$ ) and midazolam. In Uppsala propofol was used for sedation (Propolipid ${ }^{\circledR}$, Fresenius Kabi AB, Uppsala, Sweden) in the range of 0-4 mg/kg/h, and morphine (Morfin, Meda AB, Solna, Sweden) was administered as needed for analgesia.

Cerebral spinal fluid (CSF) drainage was rarely used in Cambridge, and not at all in the patients included in this study. Both Groningen and Uppsala used it fairly frequently: intermittently early, and possibly continuous drainage later.

Cambridge never used thiopental, and used decompressive craniectomies relatively frequently. Groningen and Uppsala used decompression less often than Cambridge, and sometimes used thiopental coma.

\section{Statistical Analysis}

Adherence to local CPP protocols was evaluated by computing the percentage of recorded CPP values that were within the range specified by the protocol.

We calculated the percentage of monitoring time for CPP for which a valid CPPopt curve could be computed. CPPopt curves were considered valid if they met the requirements defined by Aries et al. [9]. The mean absolute deviation of CPP from CPPopt was calculated, separately for periods above and below CPPopt and for the total duration. The percentage of CPP values more than $5 \mathrm{mmHg}$ above and below CPPopt were calculated.

To summarize the relationship between CPP and PRx one-hour averages of CPP and PRx were calculated for all patients and combined into summary CPPopt curves for each center. 
Correlations are Spearman’s R. Groups were compared using the Mann Whitney U Test. Paired comparisons were evaluated with the Wilcoxon signed rank test. The threshold for statistical significance was 0.05 .

\section{Ethical Permission}

The local ethical committees in Cambridge, Uppsala and Groningen granted permission for this study.

\section{Results}

\section{Admission and Outcome Data}

Uppsala patients tended to be older than in Cambridge or Groningen (table 1, row1). Otherwise the only significant difference was that no Groningen patients had focal injuries, compared to 10 Cambridge and 12 Uppsala patients. This was due to a policy in Groningen not to insert ICP monitors in patients with focal injuries, since they would be monitored via follow-up CT scans.

\section{Physiological parameters}

Summaries of patient physiology are shown in table 1, rows 5 - 15. ICP was well controlled in all centers. Cambridge had higher MAP values than Groningen or Uppsala. CPP was lowest in Groningen. The variability of MAP and of CPP was greatest in Uppsala. PRx was significantly lower in Groningen than in Cambridge or Uppsala.

\section{Adherence to local CPP protocols}

The percentage of monitoring time with CPP within specified ranges was computed for each patient in the study and shown as median/IQR over the patients in table 1 , rows $5-8$. Cambridge adhered to their target of CPP $\geq 60 \mathrm{mmHg} 98 \%$ (96 - 99\%) of the recorded monitoring time, and 
to their preferred target of CPP $\geq 70$ 86\% (73 - 91\%) of the time. Groningen adhered to CPP between 50 and $70 \mathrm{mmHg}$ 36\% (29 - 55\%) of the time. Uppsala adhered to CPP $\geq 60 \mathrm{mmHg}$ 97\% (93 - 99\%) of monitoring time.

\section{Last-Tier treatment}

In Cambridge 7 of the 35 patients had secondary decompressions, as did three of the 35 Groningen patients and two of the 34 Uppsala patients. Two Groningen patients and one Uppsala patient had thiopental treatment with continuous EEG monitoring.

\section{CPPopt metrics}

CPPopt was significantly lower for the Groningen patients than Cambridge patients $(\mathrm{p}<0.001$, table 2, row 1). The mean absolute CPP/CPPopt difference was greater in Uppsala than in Cambridge $(\mathrm{p}=.002)$ or Groningen $(\mathrm{p}=.003$, table 2 , row 2$)$. There were no significant differences between centers in the percentage of CPP monitoring time with a valid CPPopt (table 2, row 8).

The absolute deviation of actual CPP from CPPopt was greatest in Uppsala ( $\mathrm{p} \leq .001$, table 2, row 2). There were no significant differences between the percentages of monitoring time high-side (> CPPopt) vs low-side (< CPPopt) with CPP/CPPopt difference greater than \pm 5 mmHg, either within-center or between centers (table 2, rows 3 and 5). The magnitude of the high-side deviation in Uppsala was greater than low-side ( $\mathrm{p}=0.03$, table 2, rows 4 and 6), and also greater than high-side deviation in the other two centers (table 2, row 4). There were no between-center differences in low-side deviation (table 2, row 6).

The patients with focal injuries had a median CPPopt of 76 (73 - 82), and the diffuse group median $76(71-79)$. 


\section{Summary CPPopt graphs}

U-shaped curves with valid CPPopt values were obtained for Groningen (figure 1-A) and Cambridge (1-B), but not for Uppsala (1-C).

Summary CPPopt curves were also generated for the younger ( $<30$ years) cohorts, and older ( $\geq 50$ years) cohorts in each center (Supplemental figure 1). Valid curves were obtained for all cases except for the older patients in Cambridge (2-E) and Uppsala (2-F).

CPPopt Curves were computed for the focal and diffuse injury patient groups. These showed similar patterns with a CPPopt of 79 for the focal group, and 80 for diffuse.

\section{Admission status, physiology and outcome}

Significant correlation results are given in table 3. The only parameter we studied that was significantly correlated with outcome (GOS) was admission GCS $(\mathrm{R}=.263)$.

\section{Patient physiology and CPPopt}

Mean four-day CPPopt was highly correlated with CPP (Table 3, row 12: $\mathrm{R}=.812$ ). Correlations of CPP and CPPopt over time were also computed for each patient, with the median correlation being 0.366 (0.187 - 0.537). CPP variability (mean absolute deviation from a 4-hour moving average) was highly correlated with the absolute difference between CPP and CPPopt $(\mathrm{R}=$ 0.781). CPP variability was in turn highly correlated with MAP variability $(\mathrm{R}=.868)$, which was correlated with patient age $(\mathrm{R}=0.295)$. Older age was also associated with higher CPP, higher PRx, and greater deviation of actual CPP from optimal CPP, especially on the high side. 


\section{Discussion}

\section{Adherence to local CPP protocols}

The results presented in table 1 rows 5 - 9 show good adherence to the lower CPP thresholds. In contrast the upper limit of $70 \mathrm{mmHg}$ in the Groningen was exceeded 63\% of the time in the median case. This is probably explained by the sudden change in the 2007 BTF Guidelines, where the previous lower limit of 70 became an upper limit. Groningen follows these Guidelines. It appears that clinicians in Groningen treated the lower threshold of 50 as a hard limit, but the upper threshold as more of a suggestion. The percentage of monitoring time over 70 was significantly lower in Groningen than in Cambridge $(p<.001)$ or Uppsala $(p=.007)$, so it appears that the upper threshold did have an effect.

\section{Between-center variation in CPPopt}

CPPopt was probably lower in Groningen because CPP was lower (table 1, row 13), and CPPopt is correlated with actual CPP (table 3). CPPopt tends to follow CPP to an extent because the algorithm for estimating optimal CPP does not currently extrapolate beyond the available data.

The greater difference between CPP and CPPopt in Uppsala (table 2, row 2) is probably due to the older patient cohort (table 1, row 1). Age is positively correlated with the absolute difference between CPP and CPPopt (table 3, row 6). This is because older age is associated with increased MAP variability (table 3, row 5), which is the primary driver of CPP variability (table 3, row 9), which is in turn associated with greater deviation from CPPopt (table 3, row 11).

Aging is known to be associated with systemic hypertension and increased blood pressure variability, probably caused by stiffening of the arteries $[18,19]$. Our results suggest that in the 
older patients these factors led to less stable CPP and greater divergence from optimal CPP, especially on the high side.

Our results show very little difference between the diffuse and focal injury patient groups in the computation of CPPopt, so the absence of focal injuries in the Groningen cohort does not seem to have influenced our results.

\section{Summary CPP optimization curves}

While Groningen and Cambridge have U-shaped summary curves consistent with previous results, Uppsala shows little variation in PRx relative to CPP, and CPPopt could not be identified (figure 1). We believe that this anomaly is related to the older age of the Uppsala cohort.

In the younger patients (Supplemental figure $1 \mathrm{~A}-\mathrm{C}$ ) the results are remarkably consistent, with all three centers showing a CPPopt of about $80 \mathrm{mmHg}$. In the older patient group (Supplemental figure $1 \mathrm{D}-\mathrm{F}$ ), Cambridge and Uppsala show similar patterns with a flat, narrow range of PRx, mostly between 0.0 and 0.1 , with little dependence on $\mathrm{CPP}$, while Groningen has a clear U-shaped curve. The results for Cambridge and Uppsala suggest that changes in CPP may have less effect on ICP and cerebral hemodynamics in older patients. This could be explained by the generally stiffer cerebral arteries of older patients, that are less able to constrict and dilate in response to changes in pressure. In any case, it is evident from Supplemental figures 1-C and -F that the absence of CPPopt in the Uppsala summary (figure 1-C) is due to the preponderance of older patients. Uppsala has 21 patients in the older cohort and only 4 in the younger.

\section{Clinical Implications}

The only effect of CPP treatment on the computation or interpretation of CPPopt identified in this study was that, since CPPopt is limited to the range of observed CPP values, the effect of 
treatment on CPP tends to have a similar effect on CPPopt. Nevertheless the three centers had similar median estimates of optimal CPP, ranging from 73 to 79 mmHg (table 2, row 1 ). The small magnitude of these differences is a promising sign for the clinical utility and validity of the CPPopt methodology.

Another clinical consideration is the effect of patient age. Older patients tend to have greater divergence from CPPopt, but in many older patients the CPPopt curve flattens out, so this divergence has little effect on PRx (Supplemental figure $1 \mathrm{e}, \mathrm{f}$ ). As discussed above this is probably due to arterial stiffness, which is more prevalent in older patients.

\section{Confounding effects of age and severity of injury}

We found no correlations with outcome of age, PRx or deviation of CPP from CPPopt, possibly because in these patients age was correlated with GCS on admission $(\mathrm{R}=0.269, \mathrm{p}=0.006)$, so that older age was associated with less severe injury. Since older age was also associated with higher (worse) PRx (table 3, row 4) and with larger deviation from CPPopt (table 3, row 6), the association of older age with less severe injury may have confounded the expected correlations with outcome.

\section{Limitations of this Study}

The major limitation of this retrospective study was the lack of detailed, quantitative data regarding therapy and therapy intensity.

\section{Conclusions}

Important heterogeneity regarding CPPopt was found between centers. This may be attributed to different management protocols, especially different CPP targets, and different demographic 
factors (like age). These factors should be considered when a multicenter randomized study of optimal CPP versus fixed CPP limits trial is conducted.

\section{References}

1. Bullock R, Chesnut RM, Clifton G, et al: Guidelines for cerebral perfusion pressure. $J$ Neurotrauma 1996; 13:693-697

2. Bullock RM, Chesnut RM, Clifton GL, et al: Guidelines for cerebral perfusion pressure. $J$ Neurotrauma 2000; 17:507-511

3. Bratton SL, Chestnut RM, Ghajar J, et al: Guidelines for the management of severe traumatic brain injury 3rd edition, IX cerebral perfusion thresholds. J Neurotrauma 2007; 24:S59-S64

4. Contant CF, Valadka AB, Gopinath SP, et al: Adult respiratory distress syndrome: a complication of induced hypertension after severe head injury. J Neurosurg 2001; 95:560568

5. Carney N, Totten AM, OdReilly C, et al: Guidelines for the Management of Severe Traumatic Brain Injury, Fourth Edition. Neurosurgery 2017: 80(1):6-15 
6. Sorrentino E, Diedler J, Kasprowicz M, et al: Critical thresholds for cerebrovascular reactivity after traumatic brain injury. Neurocrit Care 2012; 16(2):258-266

7. Howells T, Elf K, Jones PA, et al: Pressure reactivity as a guide in the treatment of cerebral perfusion pressure in patients with brain trauma. $J$ Neurosurg 2005; 102(2):311-7

8. Steiner LA, Czosnyka M, Piechnik SK, et al: Continuous monitoring of cerebrovascular pressure reactivity allows determination of optimal cerebral perfusion pressure in patients with traumatic brain injury. Crit Care Med 2002; 30(4):733-8

9. Aries MJ, Czosnyka M, Budohoski KP, et al: Continuous determination of optimal cerebral perfusion pressure in traumatic brain injury. Crit Care Med 2012; 40(8):2456-63

10. Depreitere B, Güiza F, Van den Berghe G, et al: Pressure autoregulation monitoring and cerebral perfusion pressure target recommendation in patients with severe traumatic brain injury based on minute-by-minute monitoring data. J Neurosurg. 2014; 120(6):1451-7

11. Dias C, Silva MJ, Pereira E, et al: Optimal Cerebral Perfusion Pressure Management at Bedside: A Single-Center Pilot Study. Neurocrit Care 2015; 23(1):92-102 
12. Czosnyka M, Smielewski P, Kirkpatrick P, et al: Continuous assessment of the cerebral vasomotor reactivity in head injury. Neurosurgery 1997; 41:11-9

13. Steiner LA, Coles JP, Johnston AJ, et al: Assessment of cerebrovascular autoregulation in head-injured patients. A validation study. Stroke 2003; 34:2404-2409

14. Lang EW, Lagopoulos J, Griffith J, et al: Cerebral vasomotor reactivity testing in head injury: The link between pressure and flow. J Neurol Neurosurg Psychiatry 74: 1053-1059

15. Smielewski P, Czosnyka M, Steiner L, et al: ICM+: software for on-line analysis of bedside monitoring data after severe head trauma. Acta Neurochir Suppl 2005; 95:43-9

16. Timofeev I, Kirkpatrick PJ, Corteen E, et al: Decompressive craniectomy in traumatic brain injury: outcome following protocol-driven therapy. Acta Neurochir Suppl 2006; 96:11-6

17. Elf K, Nilsson P, Enblad P: Outcome after traumatic brain injury improved by an organized secondary insult program and standardized care Crit Care Med 2002; 30:2129-2134

18. Imai Y, Aihara A, Ohkubo T, et al: Factors that affect blood pressure variability. A community-based study in Ohasama, Japan. Am J Hypertens 1997; 11:1281-9 
19. Cicconetti P, Cacciafesta M, Migliori M, et al: Influence of sex and age on blood pressure variability. Arch Gerontol Geriatr 2000; 30(3):225-236 
Table 1: Inter-center comparisons shown as median and IQR of the patient distribution: P-values $<.05$ are given in the last three columns, e.g. between Cambridge and Groningen in the C-G column. GCS = admission Glasgow Coma Score; GOS = 6 month Glasgow Outcome Score. Rows 5-8 show percentage of monitoring time CPP was in specified ranges. Physiological parameters (rows 9-15) are averaged over the first four days of monitoring. MAD = mean absolute deviation from a 4-hour moving average.

\begin{tabular}{|l|l|r|r|r|r|r|r|}
\hline Row & Parameter & Cambridge & Groningen & Uppsala & C-G & C-U & G-U \\
\hline 1 & Age & $49(29-56)$ & $42(28-55)$ & $53(42-67)$ & & .015 & .012 \\
\hline 2 & Sex & $27 \mathrm{~m}, 7 \mathrm{f}$ & $26 \mathrm{~m}, 9 \mathrm{f}$ & $30 \mathrm{~m}, 4 \mathrm{f}$ & & & \\
\hline 3 & GCS & $7(3-10)$ & $6(4-8)$ & $7(7-8)$ & & & \\
\hline 4 & GOS & $3(3-4)$ & $4(3-5)$ & $3(3-5)$ & & & \\
\hline 5 & CPP $\geq 50$ & $100 \%(99-100)$ & $99 \%(98-100)$ & $100 \%(99-100)$ & .021 & & \\
\hline 6 & CPP $50-$ & $13 \%(8-26)$ & $36 \%(29-55)$ & $28 \%(21-37)$ & $<.00$ & .004 & .007 \\
& 70 & & & 1 & \\
\hline 7 & CPP $\geq 60$ & $98 \%(96-99)$ & $93 \%(85-96)$ & $97 \%(93-99)$ & $<.00$ & & .005 \\
& & & & & & \\
\hline 8 & CPP $\geq 70$ & $86 \%(73-91)$ & $63 \%(42-70)$ & $72 \%(62-80)$ & $<.00$ & .003 & .007 \\
& & & & & 1 & & \\
\hline 9 & MAP & $92(86-97)$ & $85(82-88)$ & $87(84-91)$ & $<.00$ & .024 & \\
\hline 10 & MAP MAD & $5.4(4.5-6.4)$ & $5.0(4.2-6.0)$ & $6.5(5.8-7.7)$ & & .004 & $<.001$ \\
\hline 11 & ICP & $13(11-16)$ & $14(10-17)$ & $12(9-14)$ & & & .046 \\
\hline 12 & ICP MAD & $1.8(13-2.3)$ & $1.5(1.0-2.0)$ & $1.7(1.6-1.9)$ & .026 & & .030 \\
\hline 13 & CPP & $78(74-84)$ & $72(69-74)$ & $76(73-79)$ & $<.00$ & & $<.001$ \\
& & & & & 1 & & \\
\hline 14 & CPP MAD & $2.0(1.7-2.3)$ & $1.9(1.6-2.3)$ & $2.4(2.2-2.8)$ & & .005 & $<.001$ \\
\hline 15 & PRx & $0.02(-0.04-$ & $-0.09(-0.23-$ & $0.08(-0.03-$ & .016 & & .013 \\
& & $0.18)$ & $0.11)$ & $0.13)$ & & \\
\hline
\end{tabular}


Table 2: Optimal CPP statistics: averages of minute by minute data average over the first four days. (1) CPPopt. (2) The absolute difference between CPP and CPPopt. (3) The percent of monitoring time with CPP > CPPopt +5 mmHg. (4) The absolute difference between CPP and CPPopt when CPP > CPPopt.

(5) The percent of monitoring time with CPP < CPPopt -5 mmHg. (6) The absolute difference between CPP and CPPopt when CPP $<$ CPPopt. (7) Percent of monitoring time with absolute difference between CPP and CPPopt is $<5 \mathrm{mmHg}$. (8) Percent of CPP monitoring with valid CPPopt calculated. All parameters shown as median/IQR over the patients in the study. The last three columns are as described in table 1.

\begin{tabular}{|l|l|r|r|r|r|r|r|}
\hline Row & Parameter & Cambridge & Groningen & Uppsala & C-G & C-U & G-U \\
\hline 1 & CPPopt & $79(73-$ & $73(70-$ & $75(71-$ & $<.00$ & & \\
& & $81)$ & $76)$ & $82)$ & 1 & & \\
\hline 2 & CPPopt Mean Abs Diff & $9(7-10)$ & $9(8-11)$ & $11(10-$ & & .002 & .003 \\
& & & & $13)$ & & & \\
\hline 3 & CPP > CPPopt + 5 (\%) & $33(22-$ & $26(21-$ & $30(24-$ & & & \\
\hline 4 & CPP > CPPopt Abs Diff & $8(7-11)$ & $9(8-11)$ & $11(9-13)$ & & $<.00$ & .001 \\
& & & & & & \\
\hline 5 & CPP < CPPopt - 5 (\%) & $31(19-$ & $34(27-$ & $33(26-$ & & & \\
\hline 6 & CPP < CPPopt Abs Diff & $9(7-11)$ & $9(8-11)$ & $10(8-12)$ & & & \\
\hline 7 & CPPopt Abs Diff < 5 (\%) & $36(31-$ & $37(30-$ & $30(24-$ & & & \\
& & $43)$ & $42)$ & $38)$ & & & \\
\hline 8 & CPPopt Percent Valid & $54(47-$ & $60(42-$ & $63(52-$ & & & \\
\hline
\end{tabular}


Table 3: Significant correlation results (Spearman's R). CPPopt Abs Diff is the absolute difference between CPP and CPPopt averaged over the first four days of monitoring. CPPopt Abs Diff ( $>$ ) is the same, but limited to the cases where CPP was higher than CPPopt.

\begin{tabular}{|l|l|l|r|r|r|r|}
\hline $\begin{array}{l}\text { Ro } \\
\text { W }\end{array}$ & \multicolumn{2}{|c|}{ Correlation Factors } & R & $95 \%$ Conf. & $\mathrm{R}^{2}$ & $\mathrm{P}$ \\
\hline 1 & GCS & GOS & 0.263 & $0.071-0.436$ & 0.069 & 0.008 \\
\hline 2 & Age & GCS & 0.269 & $0.080-0.440$ & 0.069 & 0.006 \\
\hline 3 & Age & CPP & 0.314 & $0.129-0.477$ & 0.100 & 0.001 \\
\hline 4 & Age & PRx & 0.309 & $0.124-0.474$ & 0.100 & 0.001 \\
\hline 5 & Age & MAP MAD & 0.295 & $0.108-0.461$ & 0.087 & 0.002 \\
\hline 6 & Age & CPPopt Abs Diff & 0.216 & $0.024-0.392$ & 0.047 & 0.028 \\
\hline 7 & Age & CPPopt Abs Diff $(>)$ & 0.240 & $0.050-0.414$ & 0.058 & 0.014 \\
\hline 8 & Age & CPPopt & 0.291 & $0.104-0.458$ & 0.085 & 0.003 \\
\hline 9 & MAP MAD & CPP MAD & 0.858 & $0.797-0.902$ & 0.736 & $<0.001$ \\
\hline 10 & ICP MAD & CPP MAD & 0.416 & $0.243-0.563$ & 0.173 & $<0.001$ \\
\hline 11 & CPP MAD & CPPopt - CPP Diff & 0.781 & $0.693-0.847$ & 0.610 & $<0.001$ \\
\hline 12 & CPP & CPPopt & 0.812 & $0.735-0.869$ & 0.660 & $<0.001$ \\
\hline
\end{tabular}



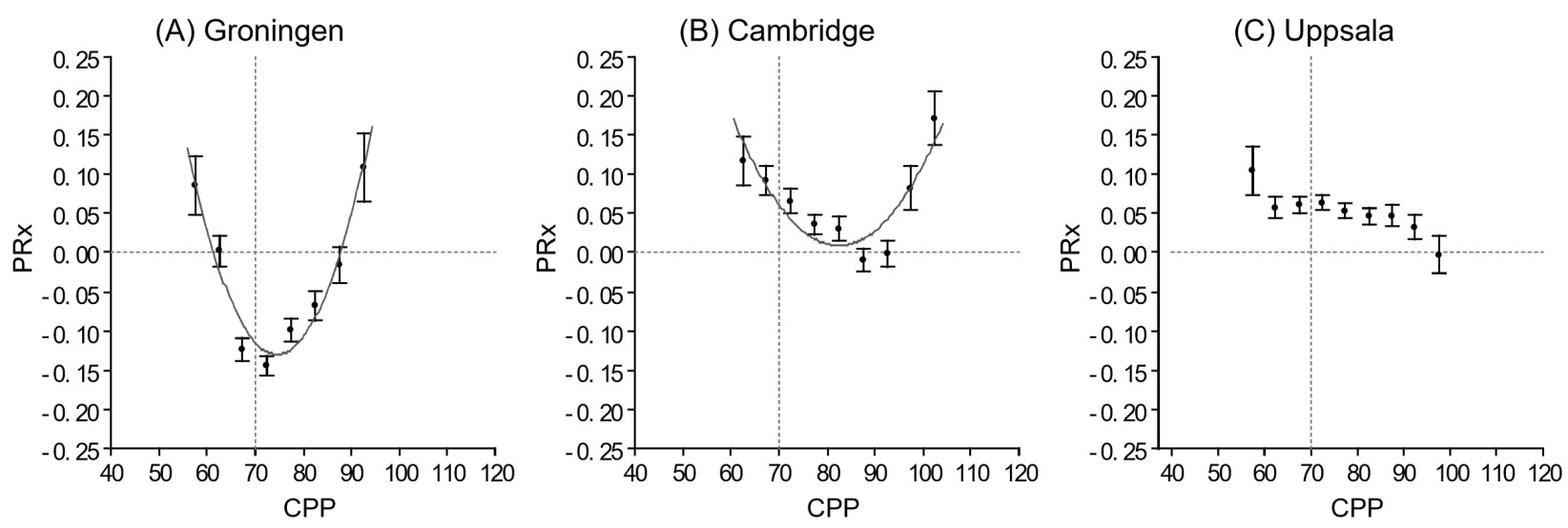

Figure 1: Summary optimization curves for the three centers. CPP/PRx paired values were computed by averaging one hour values over the first four days of monitoring. Then the CPPopt algorithm was run on the combined values for all patients in each center. CPPopt was not found in the case of the Uppsala cohort. 

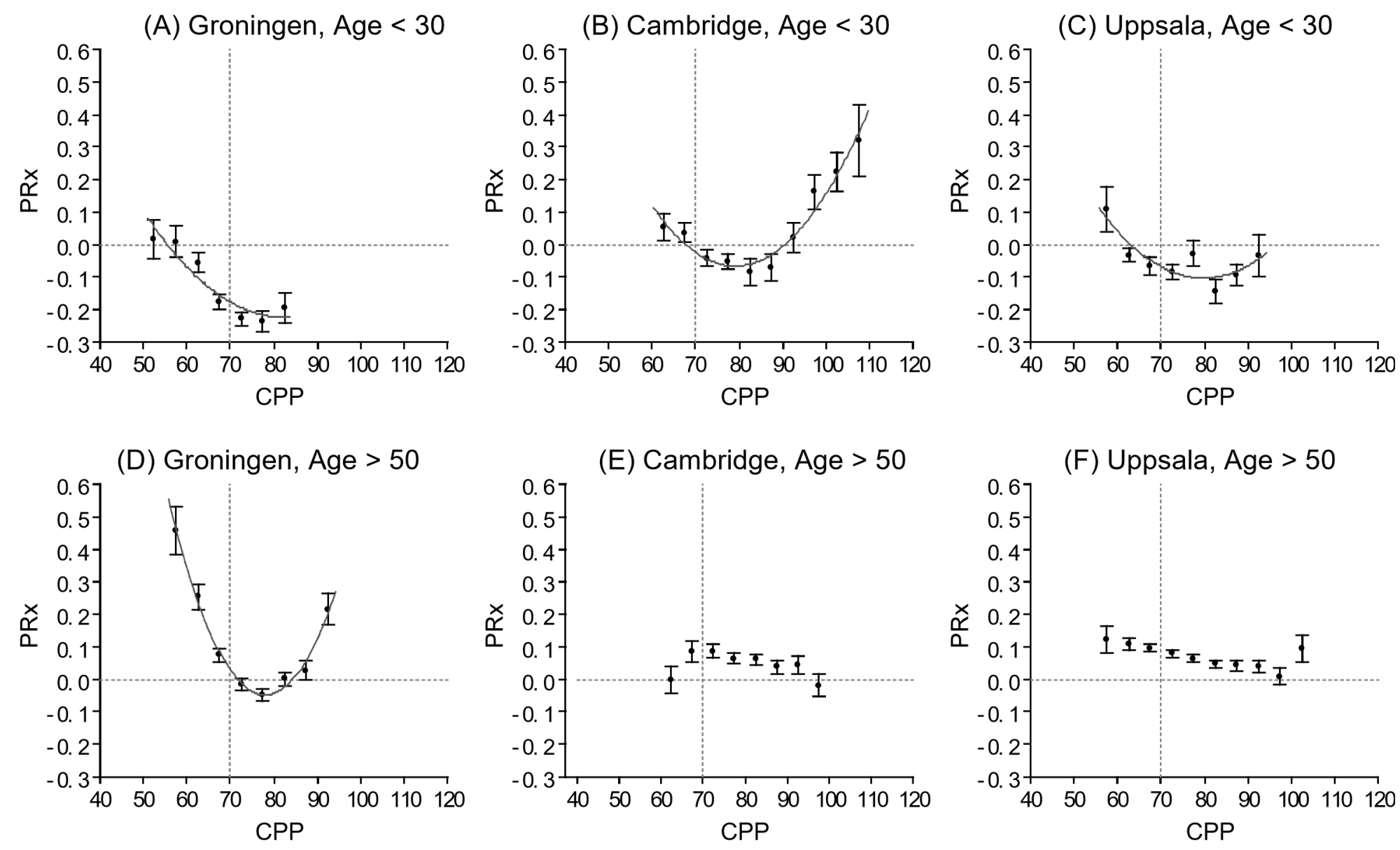

Supplemental Figure 1: The summary optimization curves for all patients in each center under the age of 30 (A-C), and 50 years and older (D-F). (A) Groningen, Young (N = 10). (B)

Cambridge, Young ( $\mathrm{N}=10)$. (C) Uppsala, Young ( $\mathrm{N}=4)$, (D) Groningen, Old ( $\mathrm{N}=14)$. (E)

Cambridge, Old ( $\mathrm{N}=15)$. (F) Uppsala, Old ( $\mathrm{N}=21)$. 